\title{
Hybridization of efficient modeling techniques for fast analysis of large-scale antenna structures in the context of the square kilometre array project
}

\author{
Citation for published version (APA): \\ Maaskant, R., Tijhuis, A. G., Mittra, R., Ivashina, M. V., Cappellen, van, W. A., \& Arts, M. J. (2008). Hybridization \\ of efficient modeling techniques for fast analysis of large-scale antenna structures in the context of the square \\ kilometre array project. In 38th European Microwave Conference, 2008 : EuMC 2008 ; 27 - 31 Oct. 2008, \\ Amsterdam, Netherlands (pp. 837-840). Institute of Electrical and Electronics Engineers. \\ https://doi.org/10.1109/EUMC.2008.4751583
}

DOI:

10.1109/EUMC.2008.4751583

Document status and date:

Published: 01/01/2008

\section{Document Version:}

Publisher's PDF, also known as Version of Record (includes final page, issue and volume numbers)

\section{Please check the document version of this publication:}

- A submitted manuscript is the version of the article upon submission and before peer-review. There can be important differences between the submitted version and the official published version of record. People interested in the research are advised to contact the author for the final version of the publication, or visit the $\mathrm{DOI}$ to the publisher's website.

- The final author version and the galley proof are versions of the publication after peer review.

- The final published version features the final layout of the paper including the volume, issue and page numbers.

Link to publication

\footnotetext{
General rights

- You may freely distribute the URL identifying the publication in the public portal. follow below link for the End User Agreement:

www.tue.nl/taverne

\section{Take down policy}

If you believe that this document breaches copyright please contact us at:

openaccess@tue.nl

providing details and we will investigate your claim.
}

Copyright and moral rights for the publications made accessible in the public portal are retained by the authors and/or other copyright owners and it is a condition of accessing publications that users recognise and abide by the legal requirements associated with these rights.

- Users may download and print one copy of any publication from the public portal for the purpose of private study or research.

- You may not further distribute the material or use it for any profit-making activity or commercial gain

If the publication is distributed under the terms of Article $25 \mathrm{fa}$ of the Dutch Copyright Act, indicated by the "Taverne" license above, please 


\title{
Hybridization of Efficient Modeling Techniques for Fast Analysis of Large-Scale Antenna Structures in the Context of the Square Kilometre Array Project
}

\author{
R. Maaskant ${ }^{1}$, A. G. Tijhuis ${ }^{\dagger 2}$, R. Mittra ${ }^{\ddagger 3}$ M. V. Ivashina ${ }^{1}$, W. A. van Cappellen ${ }^{1}$, M. J. Arts ${ }^{1}$ \\ The Netherlands institute for radio astronomy (ASTRON) \\ P.O. Box 2, 7990 AA Dwingeloo, The Netherlands \\ ${ }^{1}$ maaskanteastron.nl \\ ${ }^{\dagger}$ Faculty of Electrical Engineering, Eindhoven University of Technology \\ P.O. Box 513, $5600 \mathrm{MB}$ Eindhoven, The Netherlands \\ ${ }^{2}$ a.g.tijhuis@tue.nl \\ ${ }^{\ddagger}$ Electromagnetic Communication Laboratories, Pennsylvania State University \\ University Park, PA 16802, USA \\ ${ }^{3}$ mittradengr.psu.edu
}

\begin{abstract}
We provide an overview of the modeling techniques that have been combined to solve large-scale antenna problems within the framework of the Square Kilometre Array (SKA) project. These numerically efficient techniques have been integrated into a software tool named CAESAR ${ }^{1}$, which enables us to solve large antenna problems, both at antenna, and at system level. The latter is essential in determining the receiver sensitivity of the entire instrument, which is the main figure of merit. In the present paper we summarize how the conventional method of moments (MoM) has been enhanced using a hybridization of the Characteristic Basis Function Method (CBFM) in conjunction with a number of acceleration techniques so as to greatly reduce the overall execution time without compromising the accuracy. Representative examples are shown of realistically large and complex antenna systems that have been examined only recently.
\end{abstract}

\section{INTRODUCTION}

The Square Kilometer Array (SKA) project is a world-wide project to design and construct a revolutionary new radio telescope with a collecting area of order 1 million square meters in the wavelength range from $3 \mathrm{~m}$ to $1 \mathrm{~cm} \mathrm{[1]-[3].} \mathrm{ASTRON}{ }^{2}$ conducts the development of the aperture array concept, both by designing and examining small-scale prototype arrays, thereby demonstrating the feasibility of the instrument and revealing the potentials of various cost-effective array technologies. Concurrently, dedicated simulation software is being developed to facilitate this research phase in an accurate and time-efficient manner [4].

The antenna characterization is carried out with the aid of the method of moments (MoM), which discretizes a continuous integral equation for the unknown surface/volume current density by employing an equally large set of basis and test functions. Typically, a subsectional basis is employed to locally represent/expand the unknown current on the domain

\footnotetext{
${ }^{1}$ Computationally Advanced and Efficient Simulator for ARrays

${ }^{2}$ Netherlands institute for radio astronomy
}

that supports this vector basis function, as well as to test it using identical vector functions (Galerkin's method). The primary advantage of employing subsectional basis functions is that they can be chosen to conform to arbitrarily shaped geometries. The penalty, however, is that the size of the moment matrix becomes excessively large when a fine discretization of the corresponding integral equation for electrically large problems is required to accurately represent this current.

At present, roughly two classes of solution strategies can be identified to solve the resultant large matrix equation, either based on: (i) iterative techniques; or, (ii) iteration-free techniques. Iteration-free approaches become particularly attractive when a (direct) solution is required for a large number of excitations (MRHS). For instance, for antenna type of problems, $N$ linearly independent array excitations need to be solved to determine the full $N$-port antenna impedance matrix; hence, an iteration-free method would require $N$ matrix-vector products, whereas a standard iterative (Krylov subspace) solver may then require $\sim N^{2}$ matrix-vector products. Furthermore, by using an iteration-free approach, convergence problems are avoided that are typically associated to iterative solvers.

Iteration-free techniques rely on a strong compression of the moment matrix by employing numerically generated largedomain basis functions so that the resultant reduced matrix equation can be solved directly, e.g., by means of a standard $L U$ decomposition technique. In the Characteristic Basis Function Method (CBFM), the corresponding macro-domain basis functions (CBFs) conform to arbitrarily shaped geometries since these CBFs are constructed as fixed combinations (aggregations) of subsectional basis functions [5]. Hence, a large degree of geometrical flexibility is maintained when employing a macro basis which in itself is derived from a subsectional basis. In addition, existing computer codes can be reused/upgraded by only minor modifications. Techniques similar to CBFM are, e.g., the eigencurrent approach [6] and 
the synthetic functions approach [7].

Throughout this paper we summarize a number of numerically efficient techniques that have been used to solve a variety of SKA related problems using only moderate computing power.

\section{EXAMPLES}

All computations have been carried out in double precision arithmetic on a Dell Inspiron 9300 Notebook, equipped with an Intel Pentium-M processor operating at $1.73 \mathrm{GHz}$, and 2.0 GB of RAM.

\section{A. LOFAR LBA Station Configuration Studies Using CBFM}

With the aid of CBFM, the beam pattern of an array of 96 dual-polarized inverted-V dipole antennas (LOFAR station) has been studied for a number of different array configurations, ranging from sparse to dense, and from regular to irregular [8]. For illustration, the calculated array beam pattern $\left(\theta=30^{\circ}\right.$, $\left.\varphi=0^{0} \mathrm{scan}\right)$ is shown in Fig. 1 for one of the regular array configurations.
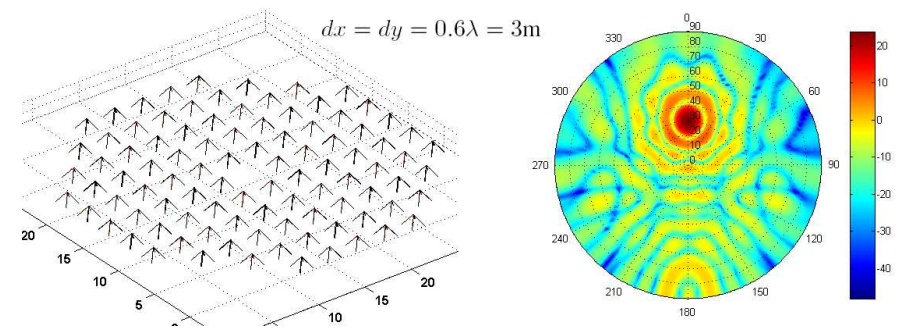

Fig. 1. Station configuration study for the LOFAR LBA project.

The antenna impedance matrix for each array configuration was computed by solving the respective reduced matrix equation

$$
\mathbf{Z}^{\text {red }} \mathbf{I}^{\text {red }}=\mathbf{V}^{\text {red }}
$$

for the unknown CBF expansion coefficient vectors $\mathbf{I}^{\text {red }}$ that belong to the equally large, though distinct, reduced excitation vectors $\mathbf{V}^{\text {red }}$ (both $\mathbf{I}^{\text {red }}$ and $\mathbf{V}^{\text {red }}$ are column-augmented matrices).

In (1), a reduced matrix block $\mathbf{Z}_{m n}^{\text {red }}$ is computed as

$$
\mathbf{Z}_{m n}^{r e d}=\mathbf{J}_{m}^{T} \mathbf{Z}_{m n}^{M o M} \mathbf{J}_{n},
$$

i.e., the fully populated moment matrix block $\mathbf{Z}_{m n}^{M o M}$, which holds the reactions between the subsectional basis/test functions supported by the $n^{t h}$ and $m^{t h}$ subdomain ${ }^{3}$, respectively, is compressed by left-right multiplication of $\mathbf{Z}_{m n}^{M o M}$ by the corresponding $n^{t h}$ and $m^{t h}$ set of CBFs $\mathbf{J}_{m}$ and $\mathbf{J}_{n}$, respectively. Each column of $\mathbf{J}_{q}$ holds the expansion coefficients of the subsectional basis representing one CBF supported by the $q^{\text {th }}$ subdomain. Superscript $T$ in (2) denotes the transposition operator.

\footnotetext{
${ }^{3}$ This domain comprises both a co- and cross-polarized element
}

Similarly, the reduced excitation vector $\mathbf{V}_{m}^{\text {red }}$ for the $m^{\text {th }}$ subdomain is computed as

$$
\mathbf{V}_{m}^{r e d}=\mathbf{J}_{m}^{T} \mathbf{V}_{m}^{M o M}
$$

Next, we compare the size of the full moment matrix (MoM) to the size of the reduced moment matrix (CBFM), as well as the corresponding total execution times. The results are listed in Table I.

TABLE I

SYSTEM MATRIX COMPRESSION AND TOTAL EXECUTION TIME.

\begin{tabular}{|c|c|c|c|c|c|}
\hline & \#RWGs & $\begin{array}{c}\text { \#MoM } \\
\text { Blocks }\end{array}$ & \#CBFs & $\begin{array}{c}\text { Matrix } \\
\text { Compression }\end{array}$ & $\begin{array}{c}\text { Total } \\
\text { Exec. Time }\end{array}$ \\
\hline MoM & 6336 & 1 & 0 & $0 \%$ & $39 \mathrm{~min} .16 \mathrm{sec}$. \\
\hline CBFM & 6336 & 179 & 768 & $98.8 \%$ & $1 \mathrm{~min} .12 \mathrm{sec}$. \\
\hline
\end{tabular}

As opposed to MoM, one observes that the execution time has dropped significantly for a CBFM approach. Also, the original moment matrix has been strongly reduced, even though the problem is relatively small, implying that the computational overhead of generating $\mathrm{CBFs}$ is relatively large.

We remark that CBFM has been used in combination with a number of additional acceleration techniques as detailed hereafter, including the exploitation of the block Toeplitz (translation) symmetry between pairs of groups of CBFs. In this respect, note that not all $96 \times 96 \mathrm{CBF}$ group interactions have to be computed to construct $\mathbf{Z}^{\text {red }}$, but only 179 as a result of the large degree of translation symmetry that a regular spaced array possesses with identical elements supporting equal sets of CBFs.

\section{B. ACA and its Solution Accuracy for the LOFAR HBA Array}

In the previous subsection, a significant acceleration has been achieved by approximating (2) as

$$
\mathbf{Z}_{m n}^{r e d} \approx \mathbf{J}_{m}^{T} \widetilde{\mathbf{Z}}_{m n}^{M o M} \mathbf{J}_{n},
$$

where $\widetilde{\mathbf{Z}}_{m n}^{M o M}$ is a low-rank approximation of $\mathbf{Z}_{m n}^{M o M}$. Matrix $\mathbf{Z}_{m n}^{M o M}$ can be constructed on-the-fly and numerically efficient using the Adaptive Cross Approximation algorithm (ACA) [9]. This is accomplished without a priori knowledge of the actual full matrix block $\mathbf{Z}_{m n}^{M o M}$. It is well-known that the effective rank becomes smaller of matrix blocks representing reaction terms between electrically distant groups of basis functions. For those groups, the rank-revealing ACA algorithm becomes highly effective, even though it will only reduce the computational complexity by a constant factor (instead of by an order).

The accuracy of the combined CBFM-ACA approach is demonstrated through an example of a $4 \times 4$ dual-polarized array of LOFAR High Band Antennas. For this purpose, the impedance and radiation characteristics, obtained by a direct MoM approach, have been overlayed with the solutions obtained by a combined CBFM-ACA approach (Fig. 2). One can observe an excellent agreement, both for the computed mutual impedances, as well as for the computed embedded 

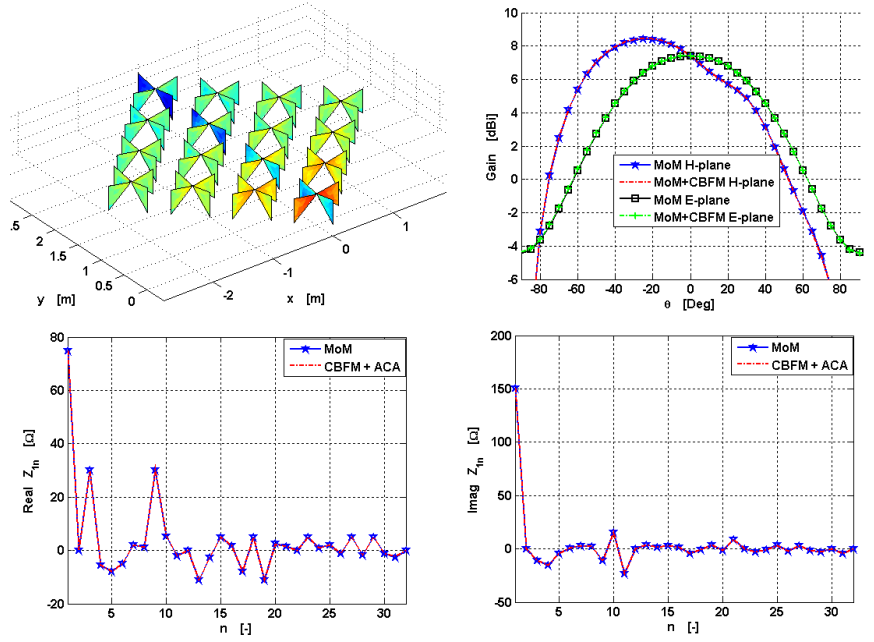

Fig. 2. Top-left: Array of LOFAR HBA antennas. Top-Right: The embedded element pattern of a corner antenna element in the E-and H-plane. BottomLeft: mutual resistance, and Bottom-Right: mutual reactance between the corner and the remaining antenna elements.

element pattern of a corner-excited HBA antenna. For this particular case, the relative error $\epsilon$ of the antenna impedance matrix $\mathbf{Z}^{\text {ant }}$ is $1.1 \%$, with the error defined as a ratio of 2 norms:

$$
\epsilon=\frac{\left\|\mathbf{Z}^{a n t}-\widetilde{\mathbf{Z}}^{a n t}\right\|^{2}}{\left\|\mathbf{Z}^{a n t}\right\|^{2}},
$$

and where $\widetilde{\mathbf{Z}}^{\text {ant }}$ is the approximated matrix.

Not surprisingly, the speed advantage of the ACA algorithm has to be traded against the solution accuracy. This tradeoff can be controlled by means of the ACA threshold [9], which directly influences the accuracy of $\widetilde{\mathbf{Z}}^{\text {ant }}$. In the present example, an ACA threshold level of 250 has been used.

\section{Arrays of Interconnected Tapered Slot Antennas}

In CBFM we decompose the entire computational domain into smaller subdomains, each of them supporting a set of CBFs. These CBFs are generated numerically, and preferably for relatively small antenna structures that have been extracted from the original antenna array, with the restriction that these small antenna structures should closely resemble their original electromagnetic array environment.

However; the generation of $\mathrm{CBF}$ becomes non-trivial for electrically interconnected (antenna) structure, since the structure is, in essence, electromagnetically inseparable. Furthermore, the CBFs have to be generated such as to synthesize a continuous current flow across the boundaries between adjacent antenna elements. A possible solution to the latter problem is to let CBFs partially overlap, or to use special bridge-functions [7], [10].

In [10] we proposed a trapezoidal post-windowing technique to generate CBFs for electrically interconnected (antenna) structures. The pertaining CBF generation procedure has been graphically visualized in Fig. 3.

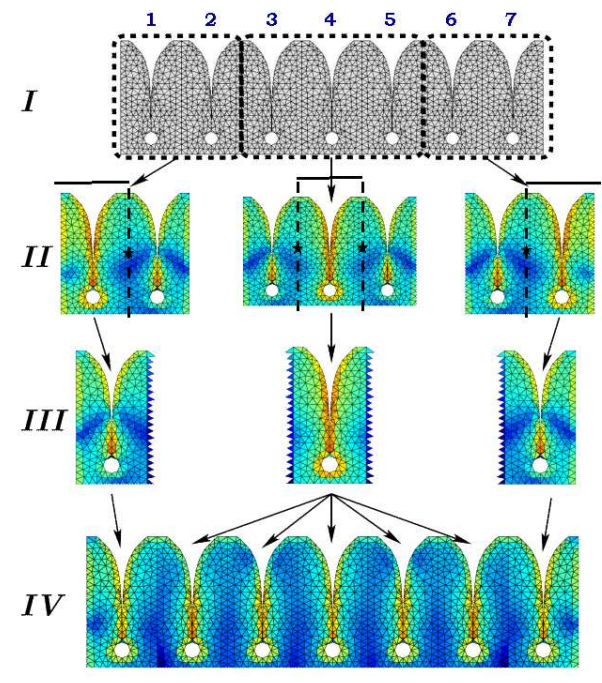

Fig. 3. Trapezoidal post-windowing technique for CBFs to synthesize currents on electrically interconnected antenna arrays.

With reference to Fig. 3, we first extract representative subarrays from the fully meshed array (Step I). Afterwards, each subarray is excited a number of times so as to generate a set of induced surface currents (Step II). Next, these currents are truncated by a trapezoidal windowing function to eliminate the undesired edge-singular currents, and to retain an overlap with adjacent domains. Finally, the so-generated CBFs (Step III) are mapped onto their corresponding array elements (Step IV). Note that, when CBFs partially overlap, they combine properly because the superposition of all taper functions sum to unity.

The aforementioned CBF windowing method has been applied to compute the impedance matrix of 576 TSA element array. Figure. 4 illustrates the array composed of 9 disjoint antenna tiles. The surface current has been synthesized using 375.000 RWG subsectional basis functions which constitute the 4320 CBFs. By exploiting translation symmetry, only 8394 out of 331776 mutual moment blocks had to be constructed, and in an time-efficient manner using the ACA algorithm. The total execution time amounted to $254 \mathrm{~min}$. $44 \mathrm{sec}$. per frequency point. The execution time can be further reduced by constructing a reduced matrix for only one of the antenna tiles while accounting for the coupling effect between the neighboring phase-steered array tiles [11].

\section{Antenna System Modeling}

In interferometric receiver systems for radio astronomy, antenna output signals are correlated to create a (steerable) beam in the sky and to achieve a certain receiver sensitivity in that direction. In practical systems, noise is generated at different stages within the receiver, and generally, microwave components are not power-matched to one another. As a result, the overall sensitivity depends upon the array receiver in its entirety.

For the purpose of analyzing the overall receiver system, a dedicated microwave simulator has been developed 


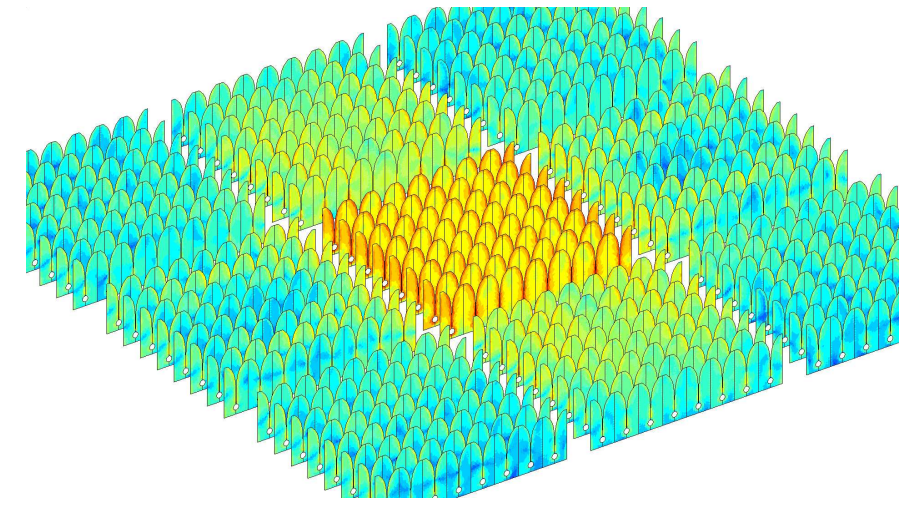

Fig. 4. Array of 9 disjoint subarrays $(3 \times 3)$, each of them composed of 64 TSA elements $(8 \times 8)$. The center tile is excited to illustrate the coupling effects. The magnitude of the surface current distribution is shown (log scale) as computed by a direct CBFM approach.

and integrated with the electromagnetic solver [12]. All microwave components, including the antenna, Low Noise Amplifiers (LNAs) and beamforming network, are characterized in terms of signal-noise wave covariance matrices, together with their scattering matrices. The cascaded compound is computed by applying the connection matrix theory as described in [14], [15].

The use of the hybridized methods enabled us to conduct a number of studies. For instance, it has been demonstrated that one has to noise match the LNAs to the active scan impedance in order to achieve minimum receiver noise in phased array antennas [13]. Another example is a microwave circuit model of a microstrip TSA feed that has been developed and combined with the antenna impedance characteristics obtained by full-wave EM simulations [16]. Figure 5 illustrates that a dielectric-free MoM code can be used to model the antenna structure separately from the feed structure.

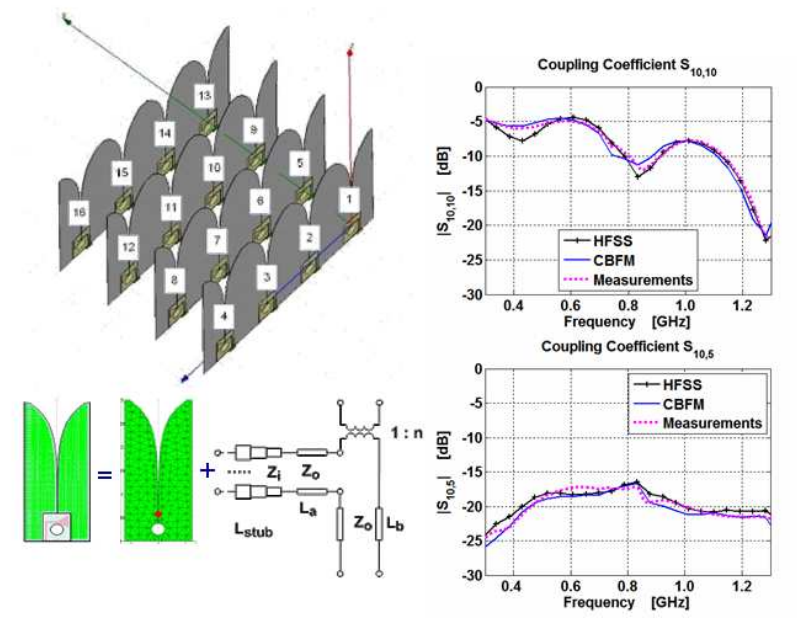

Fig. 5. Combining a microwave circuit model for the feed with a full-wave dielectric-free antenna array model.

Validation of the combined models has been performed through measurements and simulations (HFSS). A very good agreement is obtained showing that realistically complex and electrically large antenna array structures can be modeled accurately and fast.

\section{ACKNOWLEDGMENT}

The authors are grateful to both Dion Kant and Erik van der Wal (ASTRON) for their technical support on C++/Linux related problems, and Sreenivas Kasturi (UMASS) for providing us with the HFSS simulation results (Fig. 5).

\section{REFERENCES}

[1] P. J. Hall, "The square kilometre array: an engineering perspective" Reprinted from experimental astronomy, vol. 17, no. 1-3, 2004, ISBN: 1-4020-3797-x, Springer 2005.

[2] A. B. Smolders and M. P. van Haarlem, "Perspectives on radio astronomy: technologies for large antenna arrays," Conf. Proc., ASTRON, ISBN: 90805434-2-x, April 1999.

[3] Website: http://www.skatelescope.org/

[4] R. Maaskant, R. Mittra, and W. van Cappellen, "Efficient numerical analysis of prototype antennas for the Square Kilometer Array (SKA) using the Characteristic Basis Function Method (CBFM)," IEEE AP $S$ International Symposium USNC/URSI Meeting, Albuquerque, New Mexico, Jul. 9-14, 2006.

[5] V. Prakash and R. Mittra, "Characteristic basis function method: A new technique for efficient solution of method of moments matrix equations," Micr. Opt. Technol. vol. 36, pp. 95-100., Jan. 2003

[6] D. J. Bekers, S. J. L. van Eijndhoven, A. A. F. van de Ven, P. Borsboom, and A. G. Tijhuis, "Eigencurrent analysis of resonant behavior in finite antenna arrays," IEEE Trans. Microw. Theory Techn., vol. 54, no. 6, pp. 2821-2829., Jun. 2006.

[7] L. Matekovits, V. A. Laza, and G. Vecchi, "Analysis of large complex structures with the synthetic-functions approach," IEEE Trans. Antennas Propagat., vol. 55, no. 9, pp. 2509-2521, Sep. 2007.

[8] W. A. van Cappellen, S. J. Wijnholds, and J. D. Bregman, "Sparse antenna array configurations in large aperture synthesis radio telescopes," $3 r d$ European Radar Conference, pp. 76-79., Sept. 2006.

[9] R. Maaskant, R. Mittra, and A. G. Tijhuis, "Fast solution of large-scale antenna problems using the Characteristic Basis Function Method and the Adaptive Cross Approximation algorithm," submitted to IEEE Trans. Antennas Propagat., Nov. 2007.

[10] R. Maaskant, R. Mittra, and A. G. Tijhuis, "Application of trapezoidalshaped Characteristic Basis Functions to arrays of electrically interconnected antenna elements," Proc. Electromagnetics in Advanced Applications, ICEAA 2007, pp. 567-571, 17-21 Sept.

[11] R. Maaskant, R. Mittra, and A. G. Tijhuis, "Fast solving of multi-scale antenna problems for the Square Kilometre Array (SKA) radio telescope using the Characteristic Basis Function Method (CBFM) - an array of electrically large subarrays," Submitted to the ACES journal, Feb. 2008.

[12] R. Maaskant, and B. Yang, "A combined electromagnetic and microwave antenna system simulator for radio astronomy," Proc. European Conference on Antennas and Propagation (EuCAP), Nice, France, Nov. 2006.

[13] R. Maaskant, B. Woestenburg, "Applying the active antenna impedance to achieve noise match in receiving array antennas," IEEE AP-S International Symposium, Hawaii, USA, pp. 5889-5892, June 2007.

[14] J. A. Dobrowolski, "Introduction to computer methods for microwave circuit analysis and design," Artech House, Bosten, 1991.

[15] R. Maaskant, E. E. M. Woestenburg, M. J. Arts, "Generalized method of modeling the sensitivity of array antennas at system level," Proc. 34th Microwave Conference, vol. 3, pp. 1541-1544, Oct. 2004

[16] M. V. Ivashina, E. A. Redkina, and R. Maaskant, "An accurate model of a wide-band microstrip feed for slot antenna arrays," IEEE AP-S International Symposium, Hawaii, USA, pp. 1953-1956, June 2007. 